\title{
EMPYEMA THORACIS: A CURRENT PROFILE AT A TERTIARY CARE CENTER
}

\begin{abstract}
Hemanta Kumar Sethy1, Geetanjali Panda², Biswal Pradipta Trilochan³, Swetapadma Pradhan4, Gopal Krishna Sahu5,
\end{abstract} Pradeep Kumar Giri6, Dipanweeta Routray ${ }^{7}$

${ }^{1}$ Associate Professor, Department of Pulmonary Medicine, SCB Medical College and Hospital, Cuttack. ${ }^{2}$ Assistant Professor, Department of Pulmonary Medicine, SCB Medical College and Hospital, Cuttack. ${ }^{3}$ Senior Resident, Department of Pulmonary Medicine, SCB Medical College and Hospital, Cuttack. ${ }^{4}$ Senior Resident, Department of Pulmonary Medicine, SCB Medical College and Hospital, Cuttack. ${ }^{5}$ Post Graduate, Department of Pulmonary Medicine, SCB Medical College and Hospital, Cuttack. ${ }^{6}$ Senior Resident, Department of Pulmonary Medicine, SCB Medical College and Hospital, Cuttack. ${ }^{7}$ Assistant Professor, Department of Community Medicine, SCB Medical College and Hospital, Cuttack.

\section{ABSTRACT}

\section{BACKGROUND}

Empyema Thoracis (ET), the accumulation of pus in pleural cavity due to infective origin, is a perpetual clinical entity since Hippocratic era. The incidence and prevalence varies depending on different countries, type of infections, age and immune status of the host.

\section{OBJECTIVE}

To study profile of ET cases in relation to demography, clinical features, imaging, bacteriological status and treatment among patients admitted to Pulmonary Medicine Department, SCBMCH, Cuttack, Odisha.

\section{MATERIALS AND METHODS}

One hundred cases of ET were included in the study prospectively with detailed history, meticulous physical examination, necessary imaging, sputum/pleural fluid Gram and AFB staining, AFB culture and aerobic culture sensitivity followed by specific treatment.

\section{RESULTS}

Majority ET cases belonged to ages between 21-70 yrs. with relatively higher occurrence in young and middle ages, affecting more commonly males (87\%), farmers (36\%), diabetics $(22 \%)$ and alcoholics $(22 \%)$. There was no significant association of any hemithorax among tubercular empyemas (Right 49\%: Left 45\%), whereas involvement of right hemithorax was significantly higher than left in non-tubercular empyemas (Right 63\%: Left 26\%). Free ET was seen in 67\% of cases, encysted ET in 33\% cases and underlying lung parenchymal lesions in $62 \%$ cases. Pleural pus was thin in $66 \%$ cases and thick in $34 \%$ (Tubercular) cases. Gram staining of pleural fluid showed no bacteria in $82 \%$ cases, whereas it revealed growth on aerobic culture in $41 \%$ of cases. Tuberculosis was most common cause of empyema in 73\% cases (Inclusive MDR-TB 2.7\%), where Definite TB-ET was 15.1\%, Probable TB-ET $84.9 \%$ and super infection predominantly due to pseudomonas was $13.6 \%$. In contrast, Non-TB-ET was $27 \%$, in which staphylococcus aureus (33.3\%) was the major isolate followed by Ps. Aeruginosa and Esch. Coli (20\% each) on aerobic culture. Thoracocentesis was performed in 15\% cases, ICTD in $84 \%$ cases, decortication in $4 \%$ cases and Open drainage in $1 \%$ cases besides ATT and antibiotics in respective cases. In $6 \%$ cases, intrapleural fibrinolytics were given as supportive measures.

\section{CONCLUSION}

Etiological diagnosis is difficult because of prior use of antimicrobials and paucity of microorganisms following defective specimen transport/storage system. ICTD is the common treatment modality in controlling infection. Strict implementation of RNTCP DOTs is required to control most TB-ET cases in TB-prevalent areas.

\section{KEYWORDS}

Empyema, Pneumonia, Tuberculosis, BPF, Hepatic Abscess, CXR, HRCT, AFB Smear, Culture, ICTD.

HOW TO CITE THIS ARTICLE: Sethy HK, Panda G, Trilochan BP, et al. Empyema thoracis: a current profile at a tertiary care center. J. Evolution Med. Dent. Sci. 2016;5(13):547-556, DOI: 10.14260/jemds/2016/126

\section{INTRODUCTION}

Empyema Thoracis, a Latin term, is defined as pus in pleural cavity due to infective origin. This is an age-old disease well recognized since the era of Hippocrates $(600 \mathrm{BC}),[1,2]$ the first physician to describe and to treat it by surgical open drainage.[2]

Financial or Other, Competing Interest: None.

Submission 29-12-2015, Peer Review 23-01-2016,

Acceptance 30-01-2016, Published 12-02-2016.

Corresponding Author:

Dr. Hemanta Kumar Sethy,

Associate Professor,

Department of Pulmonary Medicine,

SCB MCH, Cuttack.

E-mail: sethy.drhemantakumar@gmail.com

DOI: 10.14260/jemds/2016/126
By $19^{\text {th }}$ century, various procedures like thoracocentesis, underwater seal drainage and rib resection were practiced, despite such efforts the disease proved to be fatal.[3]

In 1962, ATS described ET in 3 stages: 1) Exudative stage: in which fluid is of low viscosity allows ease expansion of lung following chest tube drainage; 2) Fibrinopurulent stage: in which there is abundant leucocytes and fibrin deposition in the visceral and parietal pleura; 3) Organizing: in which there are features of chronic inflammation and the wall of the empyema organizes with the ingrowths of capillaries and fibroblasts. Diagnosis is usually made macroscopically by purulent appearing pleural fluid during thoracocentesis and latter confirmed by smear microscopy and/or culture. Aerobic culture alone is not sufficient to determine its aetiology. 
Anaerobic and AFB cultures are very important, but they possess difficulty in collection, storage and transport system. Parapneumonic Effusion (PPE) is one of the commonest causes of ET next to tuberculosis in developing countries. Around $75 \%$ of patients with ET harbour polymicrobial organisms, whereas pus is found sterile in only $1 / 3$ rd cases.[4]

Treatment is very difficult even after diagnosis. Drug penetration into pleural cavity is impaired to achieve pleural fluid concentration. Presence of BPF (Bronchopleural Fistula) in tuberculosis takes prolonged time for closure. Delay in treatment causes various complications like empyema necessitatis, septicemia, brain abscess, amyloidosis, bronchiectasis, fibrothorax, respiratory failure and death. Early detection, diagnosis and treatment are necessary to prevent such fatal consequences.

\section{MATERIALS AND METHODS \\ Study Design}

The study was carried out prospectively, by admitting 100 cases of empyema thoracis with age ranging from 11 to $83 \mathrm{yrs}$. in Pulmonary Medicine Department, SCB MCH, Cuttack, Odisha, India, from 2012-2014.

\section{PATIENT SELECTION}

\section{Case Definition}

ET was defined as pleural effusion that fulfilled the following criteria: (1) Presence of frank pus on pleural aspiration plus (2) Presence/absence of organism on pleural fluid on Gram and AFB staining along with aerobic and AFB culture. Definite TB-ET was defined as cases of empyema showing: pleural fluid smear or culture or both positive for AFB with/without sputum smear positivity. Probable TB-ET was defined as cases: 1) Who had radiological evidence of active pulmonary tuberculosis on chest X-ray/HRCT thorax or 2) Who had shown sputum smear positive for AFB in absence of pleural fluid AFB positivity, or 3) In absence of affirmationed evidence, patients' condition improved following antitubercular therapy. Non-TB-ET was defined as cases showing pleural fluid smear and/or culture positive for bacteria without smear or culture positive for AFB. All patients were enrolled into the study after written consent and the study was carried out after clearance by ethical committee of the institution.

\section{Inclusion Criteria}

All cases of pleural effusions revealing frank pus on thoracocentesis were included in the study. Patients at all ages were encountered.

\section{Exclusion Criteria}

Those patients not giving their consent were excluded.

\section{Study Protocol}

The patients fulfilling above definition criteria were admitted to Department of Pulmonary Medicine, SCBMCH, Cuttack, Odisha and evaluated with detailed demographic and clinical parameters. Co-morbidities like diabetes mellitus, HIV infection, epilepsy, liver abscess, rheumatoid arthritis and malignancy were recorded. Meticulous physical examination and chest radiography were done in each case along with Ultrasonography and Computed Tomography of chest as per necessity. Pleural fluid samples were collected and sent for cytological analysis (DC, TLC), biochemical analysis (Protein, sugar, LDH, ADA) and microbiological study (Gram stain, aerobic culture, AFB smear for Ziehl-Neelson and fluorescent staining, AFB culture [BACTEC MGIT (Mycobacterial growth indicator tube) 960 method]. Anaerobic culture was avoided because of difficulty in collection and transport system. Complete blood counts, renal and liver profiles, HIV/HBV/HCV serology, blood sugar and sputum for AFB smear were routinely performed in all cases. In non-tubercular cases, a prolonged course of antibiotics was given according to culture sensitivity. ICTD, fibrinolytics therapy and surgical interventions were done as per need.

\section{STATISTICAL ANALYSIS}

Data was entered into Microsoft Excel and descriptive statistics analysed using the same. $\mathrm{Z}$ test and Chi square test were applied to see the statistical significant difference between two proportions and association between qualitative attributes/indicators. $P$ value $<0.05$ was taken as significant.

\section{RESULTS}

\section{Demography}

The commonest age group developing ET in our study was between 21-70 yrs. (89\%) (Fig. 1) with males outnumbering females ( $87 \%$ vs $13 \%)$. Farmers were found more frequently (36\%) to be affected than the patients with other occupations (Fig. 2).

\section{Physical Examination}

Majority of ET cases admitted with cough (83\%), fever and expectoration (78\% each), chest pain (62\%), dyspnoea (58\%) followed by malaise (41\%), loss of appetite (39\%) and haemoptysis (3\%) (Table 1). The most common physical sign was pallor in $47 \%$ cases followed by clubbing $38 \%$, pedal oedema in $24 \%$ cases. There was superficial lymphadenopathy (Cervical, axillary) only in $9 \%$ of cases, intercostal tenderness with parietal oedema in $46 \%$ cases and rib crowding in 33\% cases. Bronchial breath sound was heard in $7 \%$ cases, crackles in $17 \%$ cases and wheezes in $5 \%$ cases.

Comorbidities: Various comorbidities were associated in $70 \%$ cases, in which diabetes mellitus and alcoholism were the most common comorbidities (22\% each). There was no significant association between above two morbidities among empyema patients $\left(\mathrm{P}=0.94\right.$, Yate's $\left.\mathrm{X}^{2}=0.004\right)$ (Fig. 3).

Different aetiologies in 100 cases of ET (Table 2) Radiological manifestations of empyema thoracis and site of predilection:

Out of total 100 patients, right side involvement was in $53 \%$ cases, left side in $40 \%$ cases, but bilateral in $7 \%$ cases. There was no significant association between two hemithoraces among tubercular empyemas (R:L = 49\%: 46\%; $\mathrm{p}=0.11$ ), but right side involvement was significantly higher than the left among non-tubercular cases (R:L $=63 \%$ : 26\%, $\mathrm{p}<0.05$ ) (Table 3).

Out of all cases, $67 \%$ cases presented with free empyema and $33 \%$ cases with loculated empyema (Free empyema highly significant with $\mathrm{p}<0.05)$. Similarly uniloculated empyema was found to be highly significant than multiloculated empyema (uniloculated: multiloculated = 25/33: 8/33 with p <0.05); $29 \%$ cases of empyema were associated with pyopneumothorax and $62 \%$ of cases had underlying lung parenchymal disease, out of which PTB was $76 \%$ (47/62). (Fig. 4, 5, 6, 7) (Table 4, 5). 


\section{Thoracocentesis and Pleural Fluid Analysis}

a) Purulent appearing fluid was aspirated in all cases. The fluid was thick in $34 \%$ cases (All being tubercular) and thin in $66 \%$ cases (Both tubercular and non-tubercular). Thin fluid was significantly higher than the thick fluid $(\mathrm{Z}=3.2$, $\mathrm{p}<0.05$ ).

b) Pleural Fluid Biochemistry: Pleural fluid LDH was $\geq 1000$ $\mathrm{IU} / \mathrm{L}$ in $44 \%$ cases and $<1000 \mathrm{IU} / \mathrm{L}$ in $56 \%$ cases. Pleural fluid Glucose was $<40 \mathrm{mg} / \mathrm{dl}$ in $52 \%$ cases and $\geq 40 \mathrm{mg} / \mathrm{dl}$ in $48 \%$ of cases.

c) Microbiological characteristics of pleural fluid.

\section{Gram Staining}

Pus cells in plenty found in all cases on gram staining. Bacteria was detected in only $18 \%$ cases $(n=18)$ [Gram positive cocci $(\mathrm{n}=11)$, gram negative bacilli $(\mathrm{n}=7)]$ and no bacteria isolated in $82 \%$ cases $(n=82)$.

\section{Aerobic Culture}

Out of 100 cases, no bacterial growth was found in 59\% cases, whereas growth observed in $41 \%$ cases, out of which Gram positive bacteria (GPB) was 29\% (12/41) and Gram Negative Bacteria (GNB) was 71\% (29/41). Pseudomonas aeruginosa was the commonest species seen in $32 \%(13 / 41)$ cases followed by Staph aureus in $27 \%(11 / 41)$ cases. There was no significant difference between the proportion of empyemas showing growth and no growth $(\mathrm{p}>0.05)$, but GNB was significantly higher than GPB $(\mathrm{p}<0.05)$ (Table 6). Out of 27 cases of non-TB-ET, there was no growth in $44.4 \%(12 / 27)$ cases, but growth was isolated in 55.6\% (15/27) cases. Out of growing organisms, GPB was $40 \%(6 / 15)$ and GNB $60 \%$ (9/15). Staph aureus was most common $(33.3 \%, 5 / 15)$, followed by Pseudomonas spp. and Esch coli $(20 \%, 3 / 15$ each). There was no significant proportions between growth and no growth ( $\mathrm{p}>0.05)$. GNB was higher, but not significant in proportion to GPB ( $p>0.05)$ (Table7).

Out of 73 TB-ET cases, 35.6\% (26/73) organisms were isolated in pleural fluid on aerobic culture as superinfection. The GNB was the major isolate $(27.39 \%, 20 / 73)$ and significantly higher than GPB $(\mathrm{p}<0.05)$. Ps. aeruginosa was the commonest species found in $13.6 \%(10 / 73)$.

\section{AFB smear (ZN) Staining and Culture}

Out of 100 ET cases pleural fluid AFB smear was positive in $4 \%$ cases, AFB culture positive in $11 \%$ cases and both in $4 \%$ cases, but AFB culture was negative in $89 \%$ cases. The sensitivity of AFB smears was $5.48 \%$ and that of AFB culture was $15.07 \%$, but the specificity of both the tests were $100 \%$. Out of all 73 TB-ET cases, AFB smear was positive in $5.47 \%$ cases $(n=4)$, culture was positive in $15.1 \%$ cases $(n=11)$ and negative in $84.9 \%$ cases $(n=62)$ (Table 8$)$.

\section{Microbiological Characteristics of Sputum}

Gram Staining: Out of 100 cases of ET, Gram staining revealed gram positive cocci in $52 \%$ cases $(n=52)$, Gram negative bacilli in $3 \%$ cases and no bacteria in $29 \%$ cases.

Aerobic Culture: Out of 27 cases of non-TB -ET, no growth was observed in $89 \%(n=24)$ cases, but there were only gram negative bacterial growth in $11 \%(n=3)$ cases.(3) AFB smear staining: Out of all 100 ET cases, sputum smear positive for AFB was $8 \%(n=8)$, whereas in 73 tubercular cases, AFB smear was positive in $10.9 \%$ cases $(n=8)$.
Pleural fluid AFB smear and culture showing Definite TBET (Table 9). Out of 73 cases of TB-ET, both pleural fluid AFB smear and culture were positive in 4 cases (5.47\%), but only pleural fluid culture was positive in 7 cases (15.1\%) accounting total Definite TB-ET 15\% ( $n=11)$. Sputum and imaging showing probable TB-ET (Table 9).

Out of 73 cases of TB-ET, sputum AFB smear was positive in 8 cases $(10.9 \%)$ and only radiological shadows suggestive of tuberculosis in 41 cases (56\%) and both sputum and radiology suggesting tubercular empyema were $67 \%$ (49/73). Thirteen cases of ET $(17.8 \%, 13 / 73)$ empirically responded to anti-tubercular drugs and grouped under probable tuberculous empyema. Total probable TB-ET cases were $85 \%$ $(n=62)$.

Different aetiology responsible for non-TB-ET (Table 2). Out of 27 cases of non-TB-ET, lung abscess was found in 3 cases, pneumonia in 11 cases, liver abscess in 7 cases, post abdominal surgery in 2 cases, secondary infection due to lung mass in one case, undetermined causes responding to antibiotics in 2 cases and septicemia in one case.

\section{Treatment}

Out of 100 cases of ET, closed drainage was given in 99 cases and open drainage in one case. Out of 99 closed drainage cases, simple thoracocentesis carried out in 15 cases and ICTD (Intercostal tube drainage) given in 84 cases. Decortication was performed only in 4 cases. In 27 non-ET cases, specific antibiotics were given basing on culture and sensitivity report. In 73 TB-ET cases, CAT I was given in 57 cases (78\%), CAT II in 14 cases $(19.1 \%)$ and CAT IV in 2 cases $(2.7 \%)$.

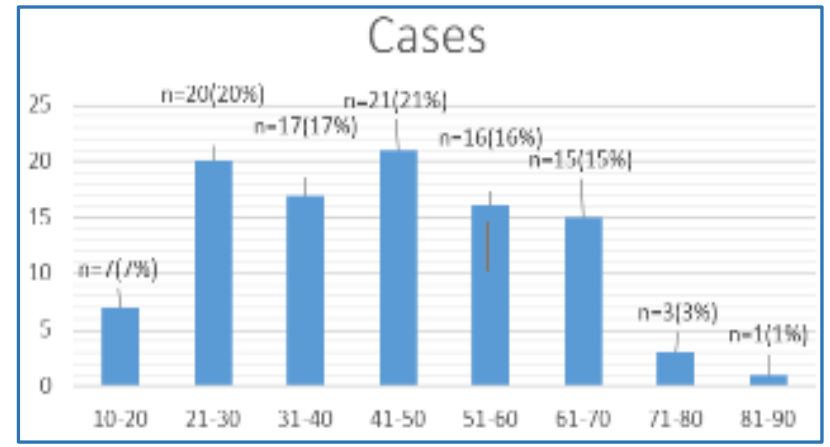

Fig. 1: Age Distribution in 100 ET patients

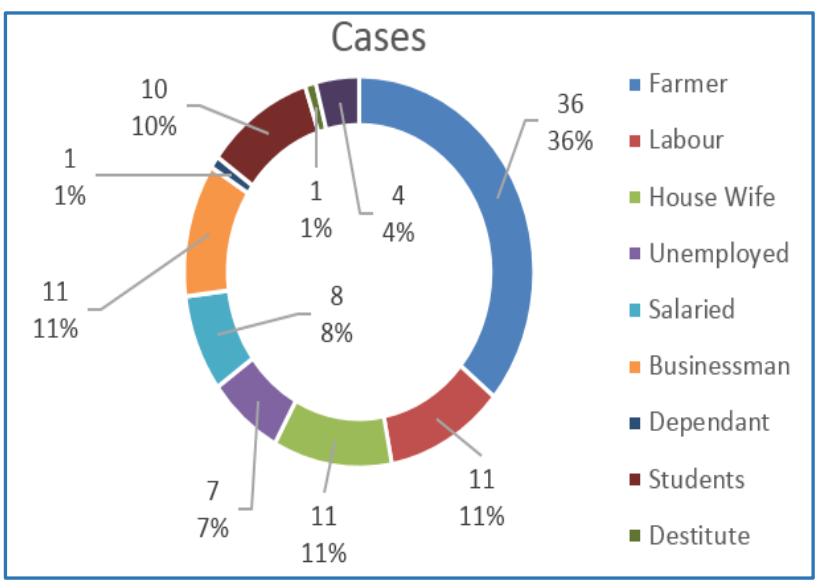

Fig. 2: Distribution of occupation in 100 ET patients 


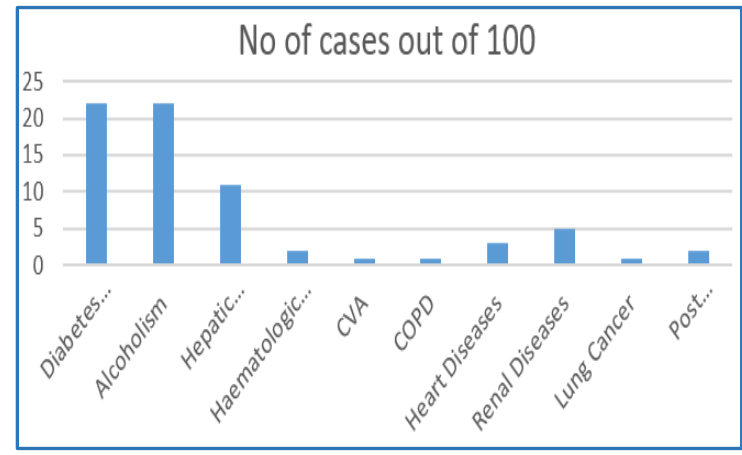

Fig. 3: Distribution of Comorbidities

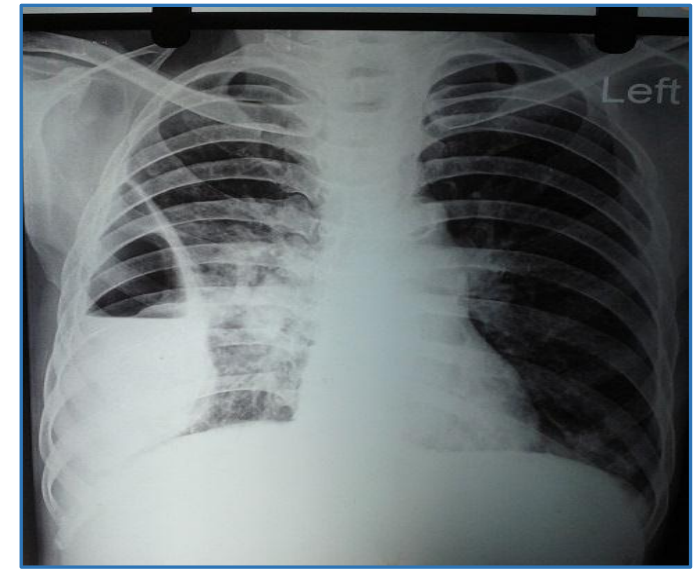

Fig. 4: Uniloculated Pyopneumothorax with underlying lung disease

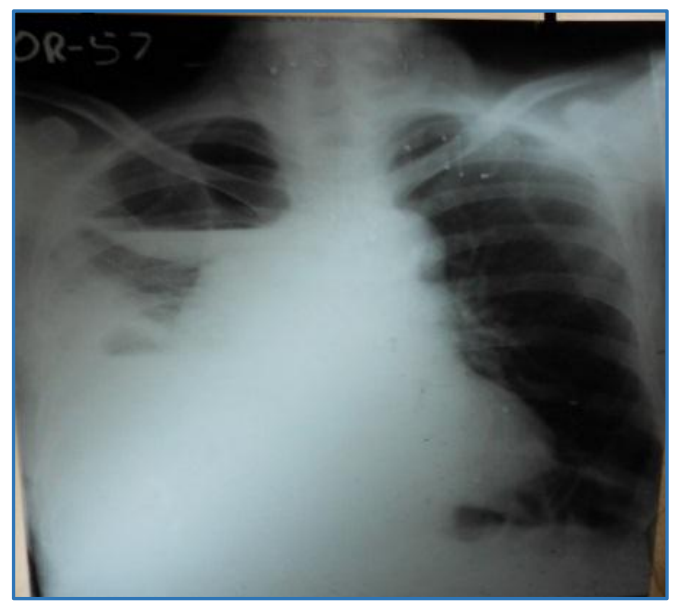

Fig. 5: CXR PA View showing

Multiloculated pyopneumothorax

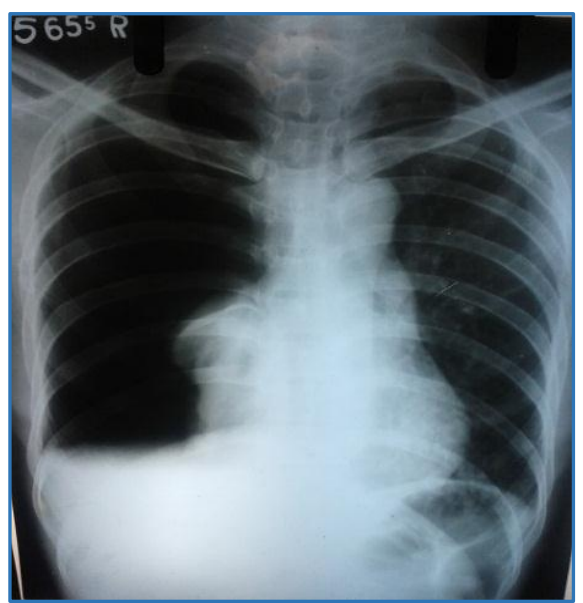

Fig. 6: CXR PA View showing Free pyopneumothorax

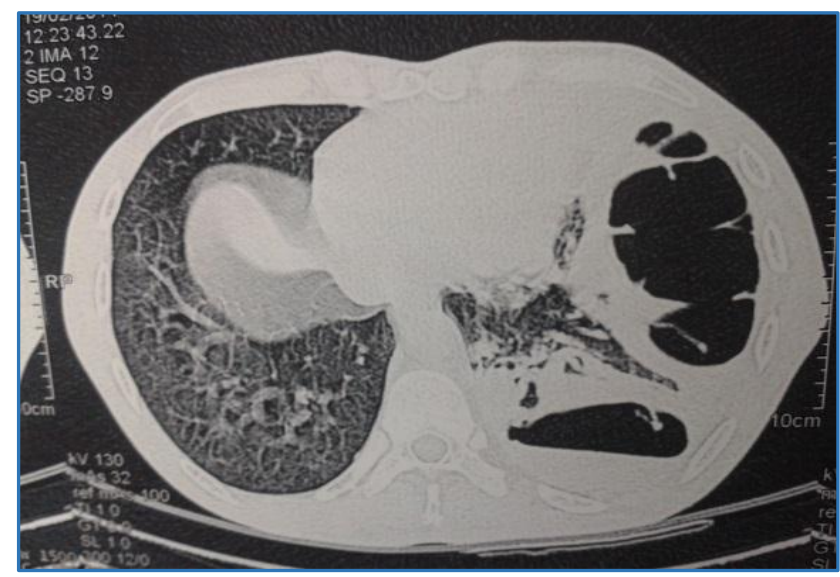

Fig. 7: HRCT Thorax showing Multiloculated pyopneumothorax

\begin{tabular}{|c|c|c|}
\hline Symptoms & $\begin{array}{l}\text { Number } \\
\text { of Cases }\end{array}$ & Percentage \\
\hline Fever & 78 & $78 \%$ \\
\hline Cough & 83 & $83 \%$ \\
\hline Expectoration & 78 & $78 \%$ \\
\hline Chest pain & 62 & $62 \%$ \\
\hline Dyspnoea & 58 & $58 \%$ \\
\hline Haemoptysis & 3 & $3 \%$ \\
\hline Malaise & 41 & $41 \%$ \\
\hline Loss of appetite & 39 & $39 \%$ \\
\hline \multicolumn{3}{|c|}{ Table 1: Presenting Symptoms in 100 ET patient } \\
\hline
\end{tabular}

\begin{tabular}{|c|c|c|}
\hline Aetiology & Number of Cases (\%) & P value \\
\hline Tubercular & $\mathbf{7 3 / 1 0 0 ~ ( 7 3 \% ) ~}$ & $<0.05^{*}$ \\
\hline Non-tubercular & $\mathbf{2 7 / 1 0 0 ( 2 7 \% )}$ & \\
Lung abscess & 3 & \\
Pneumonia & 7 & \\
Liver abscess & 1 & \\
Post abdominal surgery & 2 \\
Secondary infection in lung mass with effusion & 1 \\
\hline Septicaemia & $\mathbf{1 0 0}(\mathbf{1 0 0 \% )}$ \\
\hline
\end{tabular}

\footnotetext{
*Z test applied
} 


\begin{tabular}{|c|c|c|c|c|c|c|c|c|c|c|c|}
\hline \multirow{2}{*}{$\begin{array}{c}\text { Hemi-thorax } \\
\text { Sex } \\
\end{array}$} & \multicolumn{3}{|c|}{ Right } & \multicolumn{3}{|r|}{ Left } & & \multicolumn{3}{|c|}{ Bilateral } & \multirow[b]{2}{*}{ Total } \\
\hline & $\mathbf{M}$ & $\mathbf{F}$ & Subtotal & $\mathbf{M}$ & $\mathbf{F}$ & Subtotal & P value & M & $\mathbf{F}$ & Subtotal & \\
\hline TB & 27 & 9 & $36 / 73(49 \%)$ & 31 & 2 & $33 / 73(45 \%)$ & & 4 & 0 & $4 / 73(6 \%)$ & 73 \\
\hline Non-TB & 16 & 1 & $17 / 27(63 \%)^{*}$ & 7 & 0 & $7 / 27(26 \%)$ & $<0.05^{*}$ & 2 & 1 & $3 / 27(11 \%)$ & 27 \\
\hline Total & 43 & 10 & $53 / 100(53 \%)$ & 38 & 2 & $40 / 100(40 \%)$ & & 6 & 1 & $7 / 100(7 \%)$ & 100 \\
\hline \multicolumn{12}{|c|}{ Table 3: Hemithoracic Associations in Tb and Non-Tb in 100 cases of Empyema Thoracis } \\
\hline
\end{tabular}

*Z test applied

There was no significant association between two hemithoraces in thoracic empyemas and no significant association between hemithoraces among tubercular empyemas.

Right sided empyema was significantly higher than the left among non-tubercular cases.

\begin{tabular}{|c|c|c|c|c|c|c|c|}
\hline \multicolumn{2}{|c|}{ Types of Empyema } & Right & Left & Sub-Total & Bilateral & Total & \multirow{6}{*}{$P$ value } \\
\hline \multirow{4}{*}{ Free } & Minimal & 5 & 3 & 8 & \multirow[t]{3}{*}{5} & \multirow{3}{*}{$\begin{array}{c}13(13 \%) \\
45(45 \%) \\
20(20 \%) \\
9(9 \%)\end{array}$} & \\
\hline & $\begin{array}{c}\text { Moderate } \\
\text { (Pyopneumothorax) }\end{array}$ & $\begin{array}{c}24 \\
(10)\end{array}$ & $\begin{array}{c}21 \\
(10)\end{array}$ & $\begin{array}{c}45 \\
(20)\end{array}$ & & & \\
\hline & Massive & 3 & 6 & 9 & & & \\
\hline & Sub-total & 32 & 30 & 62 & 5 & $67(67 \%)$ & \\
\hline \multirow{3}{*}{ Loculated } & $\begin{array}{c}\text { Uniloculated } \\
\text { (Pyopneumothorax) }\end{array}$ & $\begin{array}{l}17 \\
(6)\end{array}$ & $\begin{array}{c}6 \\
6 \\
(1)\end{array}$ & $\begin{array}{l}23 \\
(7)\end{array}$ & $\begin{array}{c}2(2 \%) \\
(0)\end{array}$ & $\begin{array}{c}25(25 \%) \\
7(7 \%)\end{array}$ & \\
\hline & $\begin{array}{c}\text { Multiloculated } \\
\text { (Pyopneumothorax) }\end{array}$ & $\begin{array}{c}4 \\
(1)\end{array}$ & $\begin{array}{c}4 \\
(1)\end{array}$ & $\begin{array}{c}8 \\
(2)\end{array}$ & $\begin{array}{c}0 \\
(0)\end{array}$ & $\begin{array}{c}8(8 \%) \\
(2)(2 \%)\end{array}$ & \multirow{3}{*}{$\begin{array}{l}<0.05^{*} \\
<0.05^{*}\end{array}$} \\
\hline & Sub-total & 21 & 10 & 31 & 2 & $33(33 \%)$ & \\
\hline \multicolumn{2}{|r|}{ Total } & 53 & 40 & 93(93\%) & $7(7 \%)$ & 100 & \\
\hline \multicolumn{8}{|c|}{ Table 4: Radiological Manifestations in 100 patients with Empyema Thoracis } \\
\hline
\end{tabular}

*Z test applied.

Proportion of free empyema was highly significant than loculated empyema and that of Uniloculated empyema was highly significant than Multiloculated empyema.

\begin{tabular}{|c|c|c|}
\hline Types of Underlying Disease & No. of Cases (\%) & $<0.05^{*}$ \\
\hline Pulmonary lesions: & $62 / 100(62 \%)$ & \\
PTB & $47 / 62(76 \%)$ & \\
Pneumonia & $11 / 62(17 \%)$ & \\
Lung abscess & $1 / 62(5 \%)$ & \\
Lung Cancer & $7 / 100(7 \%)$ & \\
\hline Hepatic abscess & $\mathbf{6 9 / 1 0 0 ( 6 9 \% )}$ & \\
\hline \multicolumn{2}{|c|}{ Total } & Table 5: Underlying Parenchymal Diseases as Aetiopathogenesis In 100 ET cases
\end{tabular}

*Z test applied

Proportion of Pulmonary lesions found to be more significant than that of hepatic abscesses and proportion of PTB found to be more significant than that of pneumonia.

\begin{tabular}{|c|c|c|}
\hline Micro-organisms & Number of Cases (\%) & P value \\
\hline Growth & $41 / 100(41 \%)$ & $>0.05$ \\
\hline Gram positive bacteria & $12 / 41(29 \%)$ & $<0.05^{*}$ \\
Staph. aureus & $11 / 41(27 \%)$ & $<0.05^{*}$ \\
Strep. Pyogenes & 1 & \\
Gram negative bacteria & $29 / 41(71 \%)$ & \\
Ps. Aeruginosa & $13 / 41(32 \%)$ & \\
Kleb. Pneumoniae & 1 & \\
Proteus species & 2 & \\
Esch. Coli & $5 / 41(12 \%)$ & \\
Citrobacter species & $3 / 41(10 \%)$ & \\
Acinetobacter species & 1 & \\
Morganella morgani & Table 6: Microorganisms isolated in pleural fluid \\
\hline No growth & Aerobic culture in 100 ET cases & \\
\hline
\end{tabular}

* Z test applied. 
No significant difference between the proportions of empyemas showing growth and no-growth. But gram negative bacteria are significantly higher than gram positive bacteria.

\begin{tabular}{|c|c|c|}
\hline Microorganisms & Number of Cases (\%) & P value \\
\hline Growth & $15 / 27(55.6 \%)$ & $>0.05$ \\
\hline Gram positive bacteria & $6 / 15(40 \%)$ & \\
\hline Staph. aureus & $5 / 15(33.3 \%)$ & \\
\hline Streptococcus & $1 / 15(6.6 \%)$ & \\
\hline Gram negative bacteria & $9 / 15(60 \%)$ & \\
\hline Ps. Aeruginosa & $3 / 15(20 \%)$ & \\
\hline Proteus species & $1 / 15(6.6 \%)$ & \\
\hline Esch. Coli & $3 / 15(20 \%)$ & $\mathrm{p}>0.05^{*}$ \\
\hline Citrobacter species & $1 / 15(6.6 \%)$ & \\
\hline Acinetobacter species & $1 / 15(6.6 \%)$ & \\
\hline No growth & $12 / 27(44.4 \%)$ & \\
\hline
\end{tabular}

*Z test applied.

No significant difference seen between growth and no growth. Also no significant difference noted between proportions of gram negative bacteria and gram positive bacteria.

\begin{tabular}{|c|c|c|}
\hline Types of Investigation & $\begin{array}{c}\text { Number of cases with positivity (\%) } \\
\text { out of } \mathbf{1 0 0} \text { cases }\end{array}$ & $\begin{array}{c}\text { Number of cases with positivity out of } \\
\text { 73 cases (\%) }\end{array}$ \\
\hline AFB smears & $4(4 \%)$ & $4(5.47 \%)$ \\
\hline AFB culture & $11(11 \%)$ & $11(15.1 \%)$ \\
AFB smear and culture positive & $\begin{array}{c}4(4 \%) \\
\text { (Out of above smear and culture } \\
\text { positivity) }\end{array}$ & $4(5.47 \%)$ \\
\hline No AFB on culture & $89(89 \%)$ & $62(84.9 \%)$ \\
\hline \multicolumn{2}{|c|}{ Table 8: Pleural Fluid AFB Staining and Culture in Cases of Empyema Thoracis } \\
\hline
\end{tabular}

N.B. Sensitivity of AFB smear is $5.48 \%$ and that of AFB culture is $15.07 \%$ and specificity of both the tests are $100 \%$ among empyema patients.

\begin{tabular}{|c|c|c|c|}
\hline $\begin{array}{c}\text { Spectrum of Tubercular } \\
\text { Empyema Thoracis }\end{array}$ & $\begin{array}{c}\text { Number of cases (\%) out of 100 } \\
\text { cases of Empyema Thoracis }\end{array}$ & $\begin{array}{c}\text { Number of cases (\%) out of 73 } \\
\text { Tubercular cases }\end{array}$ & P value \\
\hline $\begin{array}{c}\text { Definite tubercular empyema } \\
\text { (PF AFB smear +ve and/or PF } \\
\text { Culture AFB +ve) }\end{array}$ & $11(11 \%)$ & $11 / 73(15.1 \%)$ \\
\hline $\begin{array}{c}\text { Probable tubercular empyema } \\
\text { (sputum AFB smear positive } \\
\text { and/or CXR positive, (n=49) } \\
\text { and positive response to ATT } \\
\text { (n=13) }\end{array}$ & $62(62 \%)$ & $62 / 73(84.9 \%)^{*}$ \\
\hline $\begin{array}{c}\text { Total tubercular empyema } \\
\text { Non-tubercular empyema } \\
\text { thoracis }\end{array}$ & $73(73 \%)$ & $<0.05^{*}$ \\
\hline \multicolumn{2}{|c|}{$27(27 \%)$} & $73(100 \%)$ \\
\hline
\end{tabular}

*Z test applied.

Proportion of Probable empyemas was significantly higher than the definite empyemas.

\begin{tabular}{|c|c|c|c|c|}
\hline \multirow[t]{2}{*}{ Authors } & \multicolumn{2}{|c|}{ PF AFB Smear Positive } & \multicolumn{2}{|c|}{ PF AFB Culture Positive } \\
\hline & $\begin{array}{c}\text { Percentage out } \\
\text { of Total cases }\end{array}$ & $\begin{array}{l}\text { Percentage out of } \\
\text { ET TB cases }\end{array}$ & $\begin{array}{c}\text { Percentage out of } \\
\text { Total cases }\end{array}$ & $\begin{array}{c}\text { Percentage out of ET TB } \\
\text { cases }\end{array}$ \\
\hline M.V. Vardhan et al. (1998) & $6 / 50(12 \%)$ & $6 / 22(27 \%)$ & $4 / 50(8 \%)$ & $4 / 22(18 \%)$ \\
\hline $\begin{array}{l}\text { Preetam Rajgopal Acharya } \\
\text { et al. (2007) }\end{array}$ & $12 / 40(30 \%)$ & $12 / 26(46 \%)$ & $6 / 40(15 \%)$ & $6 / 26(23 \%)$ \\
\hline P. Malhotra et al. (2007) & $20 / 117(17 \%)$ & $20 / 41(48 \%)$ & $16 / 117(13.6 \%)$ & $16 / 41(39 \%)$ \\
\hline $\begin{array}{l}\text { Somenath Kundu et al. } \\
(2010)\end{array}$ & $27 / 75(36 \%)$ & $27 / 29 \%(93 \%)$ & Not done & Not done \\
\hline Our study (2015) & $4 / 100(4 \%)$ & $4 / 73(5 \%)$ & $11 / 100(11 \%)$ & $11 / 73(15 \%)$ \\
\hline Table 1 & parison of $P I$ & uid Smear and & Results by Diffe & uthors \\
\hline
\end{tabular}




\section{DISCUSSION}

Presence of pus in pleural space is called ET/pleural empyema. Definition of ET varies from author to author basing on pleural fluid leukocyte count and bacterial smear/culture.

It is one of the oldest and severest of diseases. In this case, initial diagnosis is made by aspiration of frank pus, followed by identification of pathogens by microbial staining and/or culture of pleural fluid.

ET arises from various mechanisms such as direct/lymphatic extension of an infectious process within lung, hematogenous spread during septicemia from a distant focus/contamination of pleural cavity by trauma and operation/operative sequelae. Pneumonia is one of most important causes in pathogenesis of ET. About 20 to $60 \%$ of patients hospitalized for pneumonia develop PPEs and out of them 5-10\% undergo to develop ET and the mortality is high in the elderly (25-75\%).[5] Primary ET in absence of pneumonia is responsible for $4 \%$ of pleural infection. TB empyema is much less common than TB pleural effusion. Such empyema is characterized by active infection of tubercle bacilli in pleural space after cavity/lymph node abscess rupture.

Whatever the mechanisms, the resurgence of ET in the western population, is due to a combined role of the emergence of resistant organisms, nosocomial infections and increasing number of immune-compromised patients despite impact of antibiotics.[6,7] Further, in spite of high potent antitubercular drugs and their combinations, resistant cases are emerging with TB-ET. Gupta SK et al. 1989 reported: TBET represents nearly $20 \%$ of all cases of ET in high prevalent countries like India. In our study, more than $2 / 3^{\text {rd }}$ of all ET cases were TB-ET (73\%), while non-TB-ET cases were only $27 \%$.

Occupationally, majority of ET cases were farmers (36\%) followed by labours $(11 \%)$, housewives $(11 \%)$, businessmen (11\%) and students (10\%) etc.]. (Fig. 2). The incidence of ET seems to have been increasing both in children and adults worldwide. ${ }^{[8]}$ Peak incidence of ET between 21-40 yrs. age had been reported in previous studies.[9,10,11] because of more number of TB cases in India, whereas other studies.[12,13] documented higher incidence of ET after age of 40 years frequently due to non-tubercular origin in developed countries. We found maximum number of ET cases between the ages 21 to 70 yrs. with mean age 44.02 yrs. TB-ET cases with mean age 43.15 yrs. and non-TB-ET cases with mean age 46.37 yrs. in contrast to Somenath Kundu et al.[14] who reported the mean age 32.7 yrs. in tubercular group and 46.5 yrs. in non-tubercular group. The increasing age of involvement in our case specifically in tubercular cases might be due to age-related comorbidities. Higher incidence of ET in males $(87 \%)$ than in females $(13 \%)$ had also been shown in other studies. $[10,15,16]$

The symptoms in ET vary on basis of disease origin and process, lung lesions and the amount of fluid accumulated in pleural space. Cough (83\%), fever (78\%), chest pain $(62 \%)$ and dyspnea (58\%) were chief complaints in our study like other studies.[10,14,11,17] Expectoration was another major complaint found in $78 \%$ cases (Table 1) due to lung parenchymal involvement (62\%) mostly because of tuberculosis (47\%) in contrast to $52 \%$ cases as reported by M.V. Vardhan et al. (1998).[11] Hemoptysis (3\%) was a rare presentation in our study in comparison to $10 \%$ to $13 \%$ in other studies.[11,14]
Physical Signs like symptoms are also nonspecific as that can be noted in other diseases like lung abscess, pneumonia and liver abscess. In our study, the most informative signs in favour of active lesions were, i.e. stony dullness ( $100 \%$ cases), absent breath sound and intercostal tenderness $(>50 \%$ cases), parietal oedema ( $>40 \%$ cases), clubbing $(>30 \%$ cases), pedal oedema $(>20 \%$ cases), crackles $(>15 \%$ cases $)$, bronchial breath sound in ( $<10 \%$ cases $)$ and signs of chronicity i.e. pallor ( $>40 \%$ cases) and rib crowding ( $>30 \%$ cases). We observed empyema necessitans in one case and Broncho-Pleural Fistula (BPF) in $4 \%$ cases, both of tubercular origin. In contrast, Somanath Kundu et al. (2010) observed Empyema necessitans in $6 \%(n=5)$ and BPF in 25.3\% $(n=19)$ cases of tubercular origin.

Co-morbidities are very important risk factors in pathogenesis of ET. In our study, $70 \%$ of ET cases were associated with various co-morbidities in which incidences of Diabetes mellitus (22\%) and alcoholism (22\%) were more significant followed by hepatic disease (11\%). Diabetes mellitus was found to be a major co-morbidity accounting $30.7 \%(23 / 75)$ in study by Somenath Kundu et al. (2010).[14] and $17 \%$ (20/117) in study by P. Malhotra et al. (2007).[9] Alfageme and Colleagues.[18] reported a $29 \%$ incidence of alcohol abuse in ET resulting in increased incidence of anaerobic infection.

Hemithoracic Involvement depends on structural anatomy of lung airways, underlying structural lung disease, patient's posture and type of micro-organisms (Aerobic/anaerobic). In our study, there was no significant difference in involvement between two hemithoraces in tubercular empyema (Right: left $=36 \%: 33 \%$ ), whereas in non-tubercular empyema, right hemithoracic involvement was highly significant than the left $(17 \%$ vs. $7 \%)$ and that might be due to oropharyngeal aspiration through right main bronchus with subsequent pneumonia and lung abscess or direct extension from liver abscess. Right hemithoracic involvement (Right: left = 53\%:40\%) in our study might be due to higher incidence of tuberculosis in right unlike study by Asif Nadeem et al. (2004).[16]

Radiography plays pivotal role in localising the lesions, measures extension of the disease, identifies the pathologic state, suggests the aetiology and guides the treatment outcome and monitoring. We found unilateral involvement in $93 \%$ cases. There was 2 folds rise in free empyema (67\%) than loculated empyema (33\%) indicating immune status of the host. Pyopneumothorax was present in around one-third cases $(29 \%, n=29)$ representing underlying lung disease and out of these, $69 \%(n=20)$ were free and $31 \%(n=9)$ were loculated.

We detected massive empyema in $9 \%$ cases and BPF in $4 \%$ cases out of 100 cases, whereas P. Malhotra et al. (2007). [9] diagnosed massive empyema in $18.8 \%(n=22)$ cases and BPF in $41.8 \%(n=49)$ of cases following CXR. Same year Preetam Rajgopal Acharya et al. (2007).[10] in their series found free effusion in $20 \%(n=8)$ cases, air-fluid level in $75 \%(n=30)$ of cases and encysted pleural effusion in $5 \%$ of cases. The above scenario indicates the occurrence of pneumothorax seems to be decreased in our study, perhaps because of rapid expansion of DOTs and use of potential antimicrobial agents.

In our study, the underlying lung parenchymal lesions giving rise to empyema was $62 \%$ cases $(n=62)$, out of which 
PTB was $76 \%(\mathrm{n}=47)$ and non-tubercular disease was $24 \%$ $(n=15)$ in contrast to Somanath Kundu et al. (2010).[14] who reported pulmonary tuberculosis in $68.8 \%$ cases $(n=20)$. PTB was found to be highly significant than pneumonia and other pulmonary lesions in our study.

A 73\% cases of ET were of tubercular origin in our study in comparison to other studies. $[9,10,14,15]$ i.e. $35 \%, 65 \%, 38.7 \%$ and $61.97 \%$ cases respectively. Such variability might be correlated to the time period, area, criteria and methodology of the study.

Pleural fluid analysis is necessary for staging that guides initial management.[19] and therefore crucial for effective treatment. M.V. Vardhan et al. (1998).[11] found $\mathrm{pH} \leq 7.22$ in $86 \%$ of cases, pleural fluid glucose $<40 \mathrm{mg} \%$ in $44 \%$ of cases and $\mathrm{LDH}>1000 \mathrm{U} / \mathrm{L}$ in $4 \%$ of cases in their study, whereas they inserted ICT in $48 \%(24 / 50)$ cases and pleural catheter drainage in $42 \%(21 / 50)$ cases. But obtained pleural fluid glucose $<40 \mathrm{mg} \%$ in $52 \%$ of cases and LDH $>1000 \mathrm{U} / \mathrm{L}$ in $44 \%$ of cases and inserted ICTD in $84 \%$ cases, open drainage in one case and simple thoracocentesis in $15 \%$ cases. We did not estimate $\mathrm{pH}$, because of presence of pus in pleural space.

Direct isolation of bacteria from pleural fluid gives definite clue for aetiological diagnosis. Since the introduction of antibiotics, the etiological characteristics of pleural infections is changing.[2] Gram positive organisms are slowly and steadily replaced by the more resilient and resistant Gram Negative Bacteria (GNB).[20] In our study, in 100 ET cases, bacteria were found in $18 \%$ cases (Out of which, Gram +ve cocci: Gram -ve bacilli=61\%: $39 \%$ ) and no bacteria in $82 \%$ cases on gram staining.

Besides this, pleural fluid aerobic culture showed bacterial growth in $41 \%$ cases in contrast to $42 \%, 45 \%, 47 \%$, $26.6 \%$ by M.V. Vardhan et al. (1998).[11] Preetam Rajgopal Acharya et al. (2007).[10] P. Malhotra et al. (2007).[9] Somenath Kundu et al. (2010).[14] respectively. Again in 59\% cases of our study, there was no growth on aerobic culture. GNB 71\% was significantly higher than GPB 39\% $(\mathrm{p}<0.05)$, in which Ps. aeruginosa $(32 \%)$ counted majority followed by $\mathrm{S}$. aureus (27\%) (Table 6). The higher rate of GNB, i.e. $58.9 \%$ and $61.2 \%$ in pleural fluid culture was also isolated in studies by $\mathrm{P}$. Malhotra et al. (2007).[9] and Preetam Rajgopal Acharya, et al. (2007).[10] respectively. Somenath Kundu et al. (2010).[14] obtained GPB $(54.54 \%, 12 / 22)$ as the predominant organism followed by GNB 45.5\% (10/22).

Out of 27 non-TB-ET cases we found no growth in $44.4 \%$ cases in contrast to growth in $55.6 \%$ cases, out of which GNB $(60 \%)$ was the major pathogen than GPB (40\%) and S. aureus was $33.3 \%$ followed by Ps. aeruginosa/Esch. coli $20 \%$ each. The GNB was found to be higher, but not significant in proportion to GPB (Table 7). Similarly Somanath Kundu et al. (2010).[14] in their study reported pleural fluid culture positive in $43.5 \%$ cases, $\mathrm{S}$. aureus was the major pathogen (50\%) followed by Pseudomonas (13.63\%) in non-tubercular cases. The discordant results in the spectrum of pathogens causing pleural space infections had been viewed by several authors because of prior antibiotic therapy, patient specific factors (Surgical procedures, trauma or underlying conditions) or by methodological factors namely the proper specimen collection, transport and culture.[21]

We observed super-infection in 26 cases of tuberculous empyema and the most common organism being Pseudomonas aeruginosa $(n=10)$ followed by Staph. aureus $(n=6)$, whereas P. Malhotra et al. (2006).[9] observed the common organism, A. anitratus $(12.8 \%, \mathrm{n}=15)$ followed by $\mathrm{P}$. aeruginosa $(6 \%, n=7)$. Gram negative bacilli $(22.2 \%, n=26)$ were found to be the commonest organisms in their study as in our study.

Pleural fluid AFB smear is reported to be more positive in TB-ET than TB effusion (5\%). M.V. Vardhan (1998).[11] Preetam Rajgopal Acharya et al.(2007).[10] and P. Malhotra et al. (2007).[9] reported pleural fluid AFB smear positive in $27 \%$ cases, $46 \%$ cases, $48 \%$ cases and pleural fluid culture positive in $18 \%$ cases, $23 \%$ cases, $39 \%$ cases of tuberculous empyema respectively. Somenath Kundu et al. (2010).[14] reported pleural fluid AFB smear to be positive in $93.1 \%$ cases in tubercular empyema and did not proceed for pleural fluid AFB culture. But in our study, pleural fluid AFB smear was positive in $5 \%$ cases and pleural fluid culture positive in $15 \%$ cases of empyema thoracis in presence of $64 \%$ underlying PTB and 5\% cases of BPF. All authors found pleural fluid smear to be more frequently positive than pleural fluid culture, whereas our report showed very low yield in both pleural fluid smear and culture (Table 10). The low yield might be due to inhibition of TB bacillary growth in anaerobic pleural space. ${ }^{[14]}$ previous chemotherapy, collection of samples during treatment course in hospital, defective storage and transport of samples to laboratory.

It has been shown pleural fluid cultures for $M$. tuberculosis are positive in less than 40 percent of cases of pleural empyema.[22] The diagnostic yield of pleural fluid culture has been reported to be lower in solid culture media. $[22,23,24,25]$ than liquid culture media. ${ }^{[26]}$

Again, it has been reported tuberculous empyema as an extreme condition may lead to higher yield of AFB on culture due to high mycobacterial burden.[27,28] It is difficult to explain the outcome of low yield in our study in spite of use of liquid culture.

Mycobacterial culture of sputum is very helpful in suspected tuberculous pleurisy, though its sensitivity has varied widely from $0 \%$ to $52 \%$ in previous studies.[24,29] Shreng et al. 2011 reported that $48 \%$ of patients in their study had positive sputum cultures, which is comparable to previous studies by other authors.[22,24] Frequent lung parenchymal involvement in cases of tuberculous pleurisy shown in CT thorax or autopsy/thoracic surgery before advent of CT illustrated the rationality of using sputum cultures for the diagnosis of tuberculous pleurisy.[30] Therefore, the combination of effusion and sputum cultures is a reasonable approach in the diagnosis of tuberculous pleurisy and may achieve higher diagnostic yield.

We found Definite TB-ET in $15.1 \%$ of cases $(n=11)$ by considering only the pleural fluid AFB smear or culture or both positivity and found Probable TB-ET in $84.9 \%(n=62)$ cases, whereas P. Malhotra et al. (2006) reported Definite TB-ET in 48.7\% $(n=20)$ cases and Probable TB-ET in 51.2\% $(n=21)$, Somenath Kundu et al. (2010) reported Definite TB-ET in 93.1\% ( $\mathrm{n}=27)$ cases and Probable TB-ET in 6.8\% $(\mathrm{n}=2)$ case and Preetam Rajgopal Acharya et al. (2007)[10] reported Definite TB-ET in $73 \%$ of cases $(n=19)$ and Probable TB-ET in $27 \%$ of cases $(n=7)$.

Treatment modality varies from $4.7 \%-18.6 \%$ for simple aspiration and 48-95\% for ICTD as per different authors.[14, $15,16,11,10,9]$ Our study showed treatment by simple aspiration $15 \%$, ICTD $84 \%$ and open drainage $1 \%$, but the latter was 
preferred in $2 \%$ cases by M.V. Vardhan et al. (1998).[11] Decortication was performed in $80.3 \%, 21.9 \%$ and $5.2 \%$ cases in studies by Salma Ghaffar et al. (2010).[15] Asif Nadeem et al. (2004).[10] and P. Malhotra et al. (2007).[9] respectively, whereas there was $4 \%$ decortication done in our study.

We instituted ATT in 73\% TB-ET cases of empyema thoracis, in contrast to $35-65 \%$ by other studies. $[9,10,11,14,15]$ Somenath Kundu et al. (2010).[14] used CAT I DOTS in 65.5\% $(n=19)$ of cases and CAT II DOTS in $34.5 \%(n=10)$ of cases in their study, we used CAT I DOTS in $78 \%(n=57)$ of cases, CAT II in $19.1 \%(n=14)$ of cases and CAT IV in $2.7 \%(n=2)$ of cases and prolonged course of antibiotics in $27 \%$ Non-TB-ET cases in our study. Asif Nadeem et al. (2004).[10] instituted intrapleural fibrinolytics in $7.6 \%$ of cases, P. Malhotra et al. (2006) in $17 \%$ of cases, but we instituted intrapleural fibrinolytics in $6 \%$ of cases as supportive therapy.

\section{CONCLUSION}

Empyema thoracis, an age-old patient-neglected infective pleural disease, is continuing to persist in the society as a cause of severe morbidity and mortality in spite of advances in medical science. Early diagnosis is crucial for effective treatment, but not possible in early stage empyema because of sterile exudation and in latter stage due to paucity of organisms by prior antimicrobials, defective transport or local anaerobic condition not allowing bacterial growth. Treatment in late stage is also very difficult because of thick fibrin peel and multiple septations limiting effective drug penetration.

Even though molecular tests such as specific PCR for bacteria and CBNAAT for M. tuberculosis are helpful in early and rapid diagnosis, the culture sensitivity of microorganisms remains to be gold standard for its specificity in spite of poor sensitivity. Prolonged antimicrobials and continuous drainage by ICT are essential to control infection and to avoid further surgical intervention. Early presentation, detection and referral to higher centers and DOTs therapy are important components for effective management.

\section{REFERENCES}

1. Delikaris PG, Conlan AA, Abramor E, et al. Empyema thoracis--a prospective study on 73 patients. S Afr Med J 1984 Jan 14; 65(2):47-9.

2. Adam F. The Genuine Works of Hippocrates. Baltimore: Williams \& Wilkins Company, 1939; p-2.

3. Strumpell A. A text book of Medicine. London: H.K. Lewis, 1891.

4. Winterbauer RH. Non-malignant pleural effusions. In: Fishman AP, Fishman JA, Grippi MA, Kaiser LR, Senior RM, editors. Fishman's Pulmonary Diseases and Disorders. 3rd Ed. New York: McGraw-Hill; 1998. pp. 1411-28.

5. Robert B Lee: Empyema Thoracis. In Stephen C Yang et al. editors: Current Therapy in Cardiovascular and Thoracic Surgery, Mosby 2004;293-296.

6. Alfageme I, Muñoz F, Peña N, et al. Empyema of the thorax in adults. Etiology, microbiologic findings and management: Chest. 1993 Mar;103(3):839-43.

7. Delarue NC: Empyema: Principles of management-an old problem revisited. In Deslauriers et al. ed.: International Trends in General Thoracic Surgery, Vol 6. St. Louis, Mosbyu-Year Book 1990
8. Joaquín Burgos, Vicenç Falcó, Albert Pahissa, et al. Increasing incidence of empyema. Current Opinion in Pulmonary Medicine, 2013;19(4):350-356.

9. Malhotra P, Aggarwal AN, Agarwal R, et al. Clinical characteristics and outcome of empyema thoracis in 117 patients. A comparative analysis of tubercular vs nontubercular aetiologies. Respair Med 2007;101:423-30.

10. Acharya PR, Shah KV. Empyema thoracis: A clinical study. Ann Thorac Med 2007;2:14-7.

11. Vardhan MV, Tewari SC, Prasad BN, et al. Empyema thoracis-study of present day clinical and etiological profile and management techniques. Ind J Tub 1998; 45:155-60.

12. Geha AS. Pleural empyema. Changing etiologic, bacteriologic and therapeutic aspects. J Thorac Cardiovasc Surg. 1971 Apr; 61(4):626-635. [PubMed]

13. Sherman MM, Subramanian V, Berger RL. Management of thoracic empyema. Am J Surg 1977 Apr; 133(4):474479. [PubMed]

14. Kundu S, Mitra S, Mukherjee S, et al. Adult thoracic empyema: a comparative analysis of tuberculous and non-tuberculous aetiology in 75 patients; Lung India 2010;27:196-201.

15. Salma Ghaffar, Ishtiaq Ali Khan, Saida Asif, et al. Empyema thoracis: management outcome; J Ayub Med Coll Abbottabad 2010;22(3):12-17.

16. Nadeem A, Bilal A, Shah SA. Presentation and management of empyema thoracis at Lady Reading Hospital, Peshawar. J Ayub Med Coll Abbottabad 2004; 16(1):14-4.

17. Kamat. A retrospective study of 100 cases of chronic empyema in Bombay. Lung India 1985; 3:15-9.

18. Alfageme I, Munoj F, Pena, et al. Empyema in thorax in adults. Chest 1993; 103:839.

19. Steve A Sahn. Diagnosis and management of parapneumonic effusions and empyema. Clinical practice CID 2007:45 (1 December) 1480-86.

20. Chen KY, Hsueh PR, Liaw YS, et al. A 10-year experience with bacteriology of acute thoracic empyema: emphasis on Klebsiella pneumoniae in patients with diabetes mellitus. Chest 2000 Jun; 117(6):1685-9.

21. Porcel JM, Vázquez $P$, Vives $M$, et al.: Pleural space infections: microbiologic and fluid characteristics in 84 patients. The Internet Journal of Pulmonary Medicine. 2003 Volume 3 Number 1.

22. Valdes L, Alvarez D, San Jose E, et al. Tuberculous pleurisy: a study of 254 patients. Arch Intern Med 1998;158:2017-21.

23. Berger HW, Mejia E. Tuberculous pleurisy. Chest 1973;63:88-92.

24. Conde MB, Loivos AC, Rezende VM, et al. Yield of sputum induction in the diagnosis of pleural tuberculosis. Am J Respir Crit Care Med 2003;167:723-5.

25. Van de Wal BW, Wyser C, et al. Diagnostic tools in tuberculous pleurisy: a direct comparative study. Eur Respir J 2003;22:589-91.

26. Sheng Yuan Ruanetal. Revisiting tuberculous pleurisy: pleural fluid characteristics and diagnostic yield of mycobacterial culture in an endemic area. Thorax doi: 10.1136/thoraxjnl-2011-201363.

27. Bai KJ, Wu IH, Yu MC, et al. Tuberculous empyema. Respirology 1998;3:261-6. 
28. Liu SF, Liu JW, Lin MC. Characteristics of patients suffering from tuberculous pleuritis with pleural effusion, culture positive and negative for Mycobacterium tuberculosis and risk factors for fatality. Int J Tuberc Lung Dis 2005;9:1.

29. Arrington CW, Hawkins JA, Richert JH, et al. Management of undiagnosed pleural effusions in positive tuberculin reactors. Am Rev Respir Dis 1966;93:587-93.
30. Kim HJ, Lee HJ, Kwon SY, et al. The prevalence of pulmonary parenchymal tuberculosis in patients with tuberculous pleuritis. Chest 2006;129:1253-8. 